\title{
Überblick über die Bedarfsbereiche im herkunftssprachlichen Russischunterricht aus systemlinguistischer Perspektive
}

The article presents an overview of the Russian heritage language learners' learning needs resulting from the empirical research in the realm of heritage linguistics as well as from my own observations during Russian language teaching in academic courses designed for heritage speakers. The overview of the learning needs follows the description on several linguistic levels: phonological, morphological, lexical, syntactical, and on the text level.

Данная статья ставит своей задачей внести вклад в разработку специальной университетской программы по русскому языку как унаследованному. В статье предлагается обзор так называемых особых языковых потребностей или нужд (Bedarfsbereiche, learning needs) студентов с унаследованным русским языком, подобранных на основе результатов актуальных научных исследований в области эмпирической лингвистики унаследованных языков, а также на основе собственных наблюдений, возникающих в процессе преподавания русского языка в данной группе учащихся. Обзор языковых потребностей представлен на разных языковых уровнях: на фонетическом, на морфемном, на морфологическом, на лексическом, на синтаксическом уровнях и на уровне текста. 


\section{Heterogenität im universitären Russischunterricht}

Bis vor kurzem befasste sich die Fachdidaktik Russisch vorwiegend mit der Lernergruppe, die Russisch als Fremdsprache erlernt. Eine neue Lernergruppe im Russischunterricht, die die Bezeichnung HerkunftssprecherInnen (heritage speakers, zweite Migrantengeneration) trägt, bildete sich erst vor 10 bis 15 Jahren heraus. Heutzutage kann man im universitären Russischunterricht mindestens drei verschiedene Lernergruppen unterscheiden und daraus entsprechende Unterrichtstypen ableiten: Russisch als Fremdsprache, Russisch als Herkunftssprache und Russisch als zweite slawische Sprache (z.B. bei L1 Polnisch).

Jede der Gruppen weist in Hinblick auf das Erlernen der russischen Sprache ihre eigenen Bedarfsbereiche auf und erfordert von der Fachdidaktik Russisch einen differenzierten Umgang sowohl mit der Gestaltung der didaktischen Fachbücher als auch mit der Entwicklung spezieller, auf jede Gruppe zugeschnittener Curricula und Unterrichtsmaterialien. Im Folgenden wird der Fokus auf die Bedürfnisse der HerkunftssprecherInnen und die Aufgabentypen des herkunftssprachlichen Russischunterrichts gelenkt. Sowohl die einzelnen Bedarfsbereiche als auch die daraus resultierenden Aufgabentypen sind in der Forschung noch nicht zufriedenstellend erfasst.

\section{Darstellung der Bedarfsbereiche auf verschiedenen sprachli- chen Ebenen}

Die folgende überblicksartige Darstellung der einzelnen Bedarfsbereiche, die im herkunftssprachlichen Curriculum und Unterricht abgedeckt werden müssen, berücksichtigt weder eine Erwerbsprogression noch eine Er- 
werbsreihenfolge. Eher geht es hier um ihre systematische Erfassung überhaupt und nicht um die Frage, an welcher Stelle und mit welcher Komplexität und Intensität die Bedarfsbereiche in einem herkunftssprachlichen Unterricht oder in einem herkunftssprachlichen Lehrbuch eingeführt werden sollen. Eine differenzierte Darstellung der einzelnen Bedarfsbereiche, bezogen auf den Erwerb der einzelnen rezeptiven und produktiven sprachlichen Kompetenzen (Hören, Sprechen und Lesen, Schreiben) und unter Berücksichtigung der Aufgabentypologie, befindet sich in Vorbereitung (Bergmann \& Brüggemann i.V.).

Die Darstellung folgt der in der Sprachwissenschaft üblichen systematischen Beschreibung der Phänomene auf verschiedenen sprachlichen Ebenen. Die einzelnen Bedarfsbereiche werden entsprechend dargestellt auf phonetischer Ebene, auf morphematischer Ebene, auf morphologischer Ebene, auf lexikalischer Ebene, auf syntaktischer Ebene und auf der Ebene des Textes. Der Fokus der Darstellung fällt dabei auf die systemlinguistischen Bereiche. Sprachliches Handeln, Pragmatik und normatives Sprechen, interkulturelle Kompetenzen und Landes- und Kulturkunde werden hier nicht berücksichtigt, sind aber ohne Zweifel für den herkunftssprachlichen Unterricht genauso wichtig und müssen im Lernprozess entsprechend eingesetzt werden.

Voraus zu schicken ist, dass die unten dargestellten Bedarfsbereiche der phonetischen, der morphematischen und der morphologischen Ebene für den herkunftssprachlichen Schreibanfänger-Unterricht besonders wichtig sind. Sie werden aber hier aus Vollständigkeitsgründen ebenfalls kurz dargestellt (vgl. ihre Darstellung in Brüggemann 2018). Im Unterricht für Fortgeschrittene bestehen die wichtigsten Aufgaben darin, die Ausdrucksfähigkeit der konzeptionellen Schriftlichkeit zu stärken, den Erwerb des elaborierten Wortschatzes und der syntaktischen Komplexität zu fördern, sowie die bildungssprachliche Textkompetenz im Sinne einer Fähigkeit, Texte unterschiedlicher Zwecke und Strukturen selbstständig, sachbezo-

Anastasia Drackert und Katrin Bente Karl (Hg.), Didaktik der slawischen Sprachen

Beiträge zum 2. Arbeitskreis in Innsbruck (19.02.-20.02.2018)

(C) 2019 innsbruck university press, ISBN 978-3-903187-80-1, DOI 10.15203/3187-80-1 
gen und adressatenorientiert zu verfassen, zu trainieren (zur Textkompetenz bei Bilingualen vgl. Riehl 2014: 121f). Für die fortgeschrittenen HerkunftssprecherInnen sind daher die Bedarfsbereiche der lexikalischen, der syntaktischen Ebene und der Ebene des Textes von Bedeutung.

\subsection{Bedarfsbereiche auf der phonetischen Ebene}

Die Tatsache, dass viele HerkunftssprecherInnen die (konzeptionelle) Schriftlichkeit gar nicht oder nur in geringem Maße erworben haben, stört nicht zwangsläufig den Erwerb der gesprochenen Sprache. Sowohl die Aussprache als auch das spontane Sprechen können sich sehr gut entwickeln, selbst bei komplett fehlender Alphabetisierung in der Herkunftssprache. Auch wenn in der Forschung eine sehr hohe Heterogenität in den Sprachfertigkeiten der HerkunftssprecherInnen angemerkt wird (Kagan \& Polinsky 2007: 371f) - und dies betrifft ebenfalls die gesprochene Sprache -, beherrschen viele HerkunftssprecherInnen meist Betonung und Reduktion - besonders bei ihnen bekannten Wörtern - vergleichsweise gut und haben auch die Satzintonation so weit im Griff, dass der Eindruck entstehen könnte, man habe es mit einem native speaker zu tun. Handelt es sich jedoch um die Aussprache den HerkunftssprecherInnen unbekannter Wörter, kann die Reduktion außer Kraft gesetzt sein.

Wenngleich die HerkunftssprecherInnen in der spontanen Rede keine ihnen unbekannten Wörter verwenden und die phonetischen Abweichungen dadurch minimal sind, kann bei ihnen ein eigener Akzent herausgehört werden, der auf die einzelnen Ausspracheprobleme zurückzuführen ist. Damit betrifft der erste Bedarfsbereich der phonetischen Ebene die artikulatorische, praktische Phonetik und besteht in der Artikulation bzw. Rezeption einzelner Laute. Teilweise überschneiden sich die Artikulationsund Rezeptionsabweichungen der HerkunftssprecherInnen mit denen der 
FremdsprachenlernerInnen. Beide Gruppen hören keinen Unterschied z.B. zwischen stimmhaftem $\varkappa$ und stimmlosem $u$. Teilweise, besonders in Norddeutschland, tauchen artikulatorische Abweichungen auf, z.B. bei der Artikulation des Vibranten $p$ oder des lateralen $\pi$, bei der Artikulation der Vokale $u$ und $b l$. Besondere artikulatorische Aufmerksamkeit erfordern die Palatalisiertheit und Velarisiertheit des Konsonanten.

Auf der Ebene der Phonetik liegen weitere Bedarfsbereiche. Diese betreffen jedoch den Erwerb der Schriftsprache und der orthographischen Regeln und sind besonders im herkunftssprachlichen Anfänger-Unterricht relevant (Brüggemann 2018). Es scheint sinnvoll zu sein, die orthographischen Regeln im herkunftssprachlichen Unterricht nicht einfach aufzulisten und isoliert zu thematisieren, sondern über die phonetischen und morphematischen Phänomene des Russischen zu erklären und so in die Unterrichtsinhalte einfließen zu lassen. Auf diese Weise kann man die orthographischen Regeln mit den Ursachen für die sogenannte, im Russischen stark ausgeprägte, aber durchaus transparente und zum wesentlichen Teil regelbasierte Laut-Schrift-Asymmetrie verknüpfen.

Das Sich-Bewusstmachen der Laut-Schrift-Asymmetrie oder, anders gesagt, der fehlenden Phonem-Graphem-Korrespondenz - die hier als zweiter Bedarfsbereich gefasst wird - hängt mit dem Verstehen von Gesetzen der phonetischen Kombinatorik zusammen. Die fehlende PhonemGraphem-Korrespondenz wird im Russischen vor allem durch die quantitativ-qualitative Reduktion (nach harten, nach weichen Konsonanten und nach harten Zischlauten) der unbetonten Vokale verursacht, aber auch durch andere Phänomene der phonetischen Kombinatorik wie Assimilation und Stimmtonverlust. Im herkunftssprachlichen Unterricht sind daher spezielle Aufgaben zur Laut-Schrift-Asymmetrie nicht wegzudenken.

$\mathrm{Zu}$ einem der wichtigsten Bedarfsbereiche auf der phonetischen Ebene gehört der dritte: das Sich-Bewusstmachen, Wahrnehmen und Erkennen der

Anastasia Drackert und Katrin Bente Karl (Hg.), Didaktik der slawischen Sprachen

Beiträge zum 2. Arbeitskreis in Innsbruck (19.02.-20.02.2018)

(C) 2019 innsbruck university press, ISBN 978-3-903187-80-1, DOI 10.15203/3187-80-1 
Palatalisiertheit oder Velarisiertheit eines Konsonanten. Am Rande sei erwähnt, dass dieser Bedarfsbereich für die HerkunftssprecherInnen und FremdsprachenlernerInnen gleichermaßen gilt und nicht nur produktive, sondern auch rezeptive Kompetenzen betrifft. Wie beim letzten Bereich gibt es einige Verknüpfungen mit der Orthographie, z.B. die Schreibung der jotierten oder nicht jotierten Vokale nach palatalisierten und velarisierten Konsonanten oder die Regeln zur Setzung des Weichheitszeichens bzw. Härtezeichens (vgl. Brüggemann 2018).

\subsection{Bedarfsbereiche auf der morphematischen Ebene}

Blindheit für die Morphemik (und auch Morphologie), d.h. die Unfähigkeit, einer bestimmten Morphemik bestimmte Funktionen zuzuweisen und die Morphemik funktional im Text zu identifizieren und zu segmentieren, sowie die Unwissenheit gegenüber der Morphologie überhaupt machen die Morphemik zu einem weiteren Schwerpunkt im herkunftssprachlichen Russischunterricht. Daher gehört zum ersten Bedarfsbereich auf der Ebene der Morphematik das Sich-Bewusstmachen der Morphemik und des Morphem-Inventars des Russischen selbst.

Nicht nur Kenntnisse und Sich-Bewusstmachen der Morphemik, auch ihre aktive Verwendung bei der Wortbildung machen einen weiteren Schwerpunkt im herkunftssprachlichen Unterricht aus. Sinnvoll erscheinen solche Aufgaben wie z.B. gemeinsame WortfeldfamilienFindung oder Aufgaben zur Wortbildung, z.B. Bildung von Diminutiva, Bildung von desubstantivierten Adjektiven, Aufgaben zur Verbpräfigierung oder Postfigierung mit oder ohne Veränderung der lexikalischen Bedeutung, Regeln zur Interfigierung und Bildung von Komposita u. Ä. 
Ebenfalls zur morphematischen Ebene wird hier der nächste Bedarfsbereich des herkunftssprachlichen Unterrichts gezählt: das Sich-Bewusstmachen des Prinzips der Morphemkonstanz. Denn die häufigsten Abweichungen in den schriftlichen Arbeiten der HerkunftssprecherInnen basieren gerade auf der Verletzung dieses Prinzips (vgl. Brüggemann 2018).

\subsection{Bedarfsbereiche auf der morphologischen Ebene}

Die einzelnen Bedarfsbereiche auf der morphologischen (grammatischen) Ebene betreffen die oben bereits angesprochene Unwissenheit gegenüber der grammatischen Morphemik (Morphologie). In diesem Zusammenhang sind die Erkenntnisse der empirischen Herkunftssprachenforschung - zu den vollständig erworbenen, unvollständig erworbenen oder gar nicht erworbenen grammatischen Formen/Funktionen - für die Didaktikforschung von besonderer Bedeutung. Die folgende Darstellung der Bedarfsbereiche auf der morphologischen Ebene basiert auf mehreren Untersuchungen zum unvollständigen Erwerb ausgewählter grammatischer Kategorien, deren Annahmen und Ergebnisse ausführlich in Brüggemann (2016, i.V.) niedergelegt sind und hier nur in zusammengefasster Form aufgeführt werden.

Als Untersuchungsobjekte wurden die Kasus des Substantivs (ebd.: i.V.), die Kasus sowie Kurz- und Langformen des Adjektivs, die Partizipien und Passivkonstruktionen ausgewählt (ebd.: 2016). Es wurden zwei Testformate angewendet: die Kasus des Substantivs und des Adjektivs wurden in einem Lückentest erfragt, andere Kategorien im Grammatikalitätsurteilstest (GU-Test) erhoben. Im GU-Test wurden die Testsätze nicht nur zur Beurteilung vorgelegt, sondern die ProbandInnen mussten die Sätze auch

Anastasia Drackert und Katrin Bente Karl (Hg.), Didaktik der slawischen Sprachen

Beiträge zum 2. Arbeitskreis in Innsbruck (19.02.-20.02.2018)

(C) 2019 innsbruck university press, ISBN 978-3-903187-80-1, DOI 10.15203/3187-80-1 
korrigieren. Damit konnte überprüft werden, ob die ProbandInnen tatsächlich die erforderlichen Stellen als falsch beurteilen. An diesen Untersuchungen nahmen nur diejenigen ProbandInnen teil, die entweder in Deutschland geboren sind und Russisch als Sprache der innerfamiliären Kommunikation haben oder im Alter bis 7 Jahre - vor der Einschulung in Russland also - mit ihren Eltern immigriert sind. Außerdem haben diese ProbandInnen vor dem Beginn des Slawistikstudiums keinen gesteuerten Russischunterricht erhalten. Es ist also eine Gruppe, deren RussischKenntnisse rein auf den mündlichen Spracherwerb, auf den Erwerb der gesprochenen Sprache zurückzuführen sind und deren schriftsprachliche und grammatische Kompetenzen noch nicht systematisch im gesteuerten Russischunterricht trainiert wurden. Zu Beginn der Untersuchung haben die ProbandInnen gerade im universitären Sprachunterricht das Alphabet gelernt und konnten mühsam Lese- bzw. erste Schreibversuche unternehmen.

Die zentrale Annahme der beiden Untersuchungen ist, dass der rein mündlich geprägte Spracherwerb den ungesteuerten, natürlichen Erwerb der grammatischen Kategorien maßgeblich beeinflusst. Er hat zur Folge, dass in der Herkunftssprache nur diejenigen grammatischen Kategorien erworben werden können, die in der gesprochenen russischen Sprache zu finden, genauer gesagt zu hören sind. ${ }^{1}$ Daher bildet die mündlich geprägte Morphologie (aber auch andere mündlich geprägte sprachliche Bereiche: Morphemik, Syntax, Lexik, Textebene) eine Art Basis für den unvollständigen herkunftssprachlichen Spracherwerb (zur Syntax vgl. Kukla i.V.).

In der oben erwähnten Untersuchung hat sich die Annahme bestätigt, dass die in der gesprochenen Sprache fehlenden grammatischen Strukturen

\footnotetext{
${ }^{1}$ Erwähnt seien in diesem Zusammenhang zahlreiche Untersuchungen zur gesprochenen Sprache oder zur russischen Umgangssprache von Zemskaja u.a. (Zemskaja 1973, 1981, 1983), in denen nicht nur die Besonderheiten der gesprochenen Sprache, sondern auch konkrete sprachliche Teilbereiche wie die mündlich geprägte Morphemik, mündlich geprägte Morphologie und mündlich geprägte Syntax und Textsorten diskutiert werden.
} 
auch in der Herkunftssprache fehlen bzw. von den HerkunftssprecherInnen nicht erkannt und akzeptiert werden. Es ist also davon auszugehen, dass bestimmte grammatische Kategorien oder bestimmte Bereiche der Kategorien nicht vollständig sind oder nicht als stabil bzw. als mehr oder weniger stabil, einzustufen sind. Wenn wir zur Frage nach den Bedarfsbereichen im herkunftssprachlichen Unterricht zurückkommen, kann man die einzelnen Bedarfsbereiche auf der morphologischen Ebene mit der „Stabilisierung" oder dem Erwerb der in der Herkunftssprache fehlenden oder unvollständig erworbenen Kategorien zusammenbringen.

In der Untersuchung der Substantivmorphologie konnte gezeigt werden, welche einzelnen Subkategorien besonders instabil sind (Dativ, Präpositiv, Instrumental im Plural, mit jotierten Graphemen). Konkret bedeutet das, dass die Endungen - $a м,-a x,-a м u$ und vor allem die den palatalisierten Konsonanten folgenden jotierten Endungen - $я м$, $-я x$ und $-я м u$, wie z.B. in преподавателями 'Lehrer.MASK.PL.INSTR.' - also die Endungen aus mehreren Buchstaben - als unvollständig oder als nicht erworben gelten. Vor allem aber ging daraus hervor, dass (In-)Stabilität ein mehrdimensionales Konstrukt ist, auf das mehrere Faktoren einwirken. Sie ist ein bewegliches Phänomen, d. h. ein und dieselbe Endung wird bei einem häufig in der gesprochenen Sprache vorkommenden Lexem von HerkunftssprecherInnen richtig eingesetzt, bei einem weniger häufigen, schriftsprachlichen Lexem jedoch nicht. Interessanterweise spiegelt sich in der Untersuchung bis auf den Dativ die Häufigkeitsreihenfolge von Kasus in der russischen gesprochenen Sprache von Zemskaja (1983) wider. ${ }^{2}$ Auf die Instabilität des Instrumentals bei den in Amerika lebenden HerkunftssprecherInnen weist Polinsky hin (1997: 375).

\footnotetext{
${ }^{2}$ Zemskaja (1983: 139) stellt folgende Reihenfolge fest: Nominativ (32,5\%), Akkusativ $(25,5 \%)$, Genitiv (22\%), Präpositiv (11\%), Instrumental (5\%), Dativ (4,1\%). In der Wissenschaftssprache steht dagegen der Genitiv an der ersten Stelle.
} 
Im Bereich der Adjektivkasus nimmt die Stabilität der Endungen im obliquen Kasus deutlich ab. Die Adjektivendungen werden also kaum beherrscht, und insbesondere der Instrumental erweist sich als eine instabile Kasussubkategorie des Adjektivs. Diese starke Instabilität der Adjektivendungen kann damit erklärt werden, was Zemskaja (1983: 139f) hinsichtlich der Adjektive in der russischen gesprochenen Sprache anmerkt: Die Adjektive seien in der russischen Umgangssprache äußerst selten und würden fast ausschließlich in der prädikativen Funktion gebraucht (also als Prädikatsnomen und im Nominativ, z.B. statt Встань с грязного пола! 'Steh vom dreckigen.MASK.SG.GEN. Boden auf!' wird in der gesprochenen Sprache die Konstruktion Встань! Пол же грязный! 'Steh auf! Der Boden ist doch dreckig.MASK.SG.NOM.!' präferiert). Mit der niedrigen Frequenz der Adjektive in obliquen Kasus in der gesprochenen Sprache kann die niedrige Stabilität des obliquen Adjektivkasus in der Herkunftssprache begründet werden.

Nicht überraschend waren die Ergebnisse des GU-Tests hinsichtlich der Adjektivkategorie Kurz- und Langform: Die Kurzformen wurden im Test zu 66\% abgelehnt und meist mit den Langformen korrigiert, z.B. der Satz Учитель был добр 'Der Lehrer war gut (nett).MASK.SG.NOM.KF.' wurde korrigiert durch Учитель был добрый 'Der Lehrer war gut (nett).MASK.SG.NOM.LF.'. Die in der russischen gesprochenen Sprache seltenen Kurzformen (vgl. dazu Zemskaja 1973: 199f) bilden also in der Herkunftssprache eine weitere instabile Adjektivkategorie.

Parallelen zwischen der Morphologie der Herkunftssprache und der der russischen gesprochenen Sprache können ebenfalls beim Verb gezogen werden. Passiv-Sätze wurden von den HerkunftssprecherInnen zu 58\% als nicht korrekt eingestuft. Insbesondere das -sja-Passiv von imperfektiven Verben, wie z.B. читается 'wird gelesen' wurde mehrheitlich abgelehnt und ist als eine instabile Kategorie anzusehen. Dagegen erwies sich das -n-/-t-Passiv, wie z.B. прочитана 'wurde gelesen' als relativ stabil (mit 
68\% als korrekt eingestuft). Dies entspricht der Feststellung von Krasil'nikova (in Zemskaja 1973: 160ff), dass bei den Verbparadigmen in der russischen Umgangssprache (gesprochenen Sprache) nur finite Formen, wenige Passivsätze, aber - $n-/-t$-Formen in der Tempusfunktion Perfekt, wie z.B. У меня билеты заказаны 'Ich habe die Karten bestellt' zu finden sind. Entsprechend schnitten die Partizipialadjektive bis auf die - $n-/-t$-Formen als relativ unstabil $\mathrm{ab}$, während sich die -n-/t-Form, wie z.B. прочитанная статья 'der gelesene Aufsatz', dagegen als stabil erwies, sodass selbst nicht existierende -n-/-t-Bildungen, wie z.B. *погуленный $n \ddot{e} c$ '*der spazierengegangene Hund' mit durchschnittlich $48 \%$ als richtig beurteilt wurden.

An dieser Stelle ist noch anzumerken, dass die grammatische Stabilität der Kategorien in der Herkunftssprache Russisch weiterer tiefgehender Untersuchungen bedarf, in denen solche Faktoren wie lexikalische Häufigkeit, stilistische Zugehörigkeit oder lexikalische Konkretheit/Abstraktheit eines Wortes, korpusbasierte Häufigkeit der Form u.a. berücksichtigt werden.

\subsection{Bedarfsbereiche auf der lexikalischen Ebene}

Die lexikalische Beschaffenheit der Herkunftssprache Russisch wurde in den Arbeiten von Anstatt (2011) und Karl (2012) untersucht. Allerdings fokussiert die erste Arbeit primär auf den Aspekt der lexikalischen Attrition bei der Sprachproduktion (nicht Rezeption) im Zusammenhang mit der Wortfrequenz und die zweite Arbeit auf den Bereich der semantischen Transferenz aus der Umgebungssprache Deutsch (dazu unten). Fragen nach dem unvollständigen Erwerb des Wortschatzes und nach der lexikalischen (In-)Stabilität der Herkunftssprache Russisch im Zusammenhang mit dem mündlich geprägten Spracherwerb wurden noch nicht erforscht.

Anastasia Drackert und Katrin Bente Karl (Hg.), Didaktik der slawischen Sprachen

Beiträge zum 2. Arbeitskreis in Innsbruck (19.02.-20.02.2018)

(C) 2019 innsbruck university press, ISBN 978-3-903187-80-1, DOI 10.15203/3187-80-1 
Ähnlich wie beim unvollständigen Erwerb der morphologischen Kategorien wird hier beim Erwerb des Wortschatzes angenommen, dass der mündliche Erwerbsmodus nur die Lexik-Schicht aus der gesprochenen Sprache mit sich bringt. Die Lexik der konzeptionellen Schriftlichkeit bleibt weitgehend unerworben bzw. ist als instabile Schicht anzusehen. Z.B. kann die schriftsprachliche Lexik weitgehend rezeptiv erkannt, kaum aber bei der Sprachproduktion abgerufen werden. Weiterhin bleibt die instabile Lexik-Schicht hinsichtlich ihrer lexikalischen Bedeutung nicht „treffsicher“, d.h. die Bedeutungen können von HerkunftssprecherInnen anders oder abweichend identifiziert werden, z.B. semantisch über- oder unterdiskriminiert oder hinsichtlich der selektiven Restriktion verändert gebraucht werden, als dies in der Norm der Fall ist.

Stabil ist dagegen der Bereich der Lexik, der auf die Alltagskommunikation zu Hause zurückzuführen ist, z.B. aus den Bereichen Essen, Wohnung, Körperpflege, Kleidung, Spielzeug und Schulsachen. Interessanterweise bleibt der stabile lexikalische Bereich als gesamter Wortschatz über die Jahre bestehen, sodass die heranwachsenden HerkunftssprecherInnen in den Selbsteinschätzungen in nicht dokumentierten Einstufungsgesprächen ihren Wortschatz als „eingefroren“ (auf der Ebene eines Vorschulkindes) beschreiben. Ab der Einschulung wird systematisch an der Erweiterung des deutschen Wortschatzes gearbeitet, während der russische Wortschatz ohne gesteuerten Unterricht auf der Ebene eines Vorschulkindes bleibt. Überhaupt hat die herkunftssprachliche Lexik viele kindersprachliche Elemente, solche z.B. wie Diminutive: statt kniga 'Buch' oder podruga 'Freundin' präferieren die Herkunftssprecher knižka '(kleines, nicht wissenschaftliches) Buch' (*knižka Apresjana) oder podružka '(kleine) Freundin'. Wenn man sie darum bittet, z.B. das Wort portfel' zu übersetzen, ist das Resultat eher 'Schulranzen' als 'Aktenkoffer'.

Bekanntlich ist der lexikalische Bereich einer Sprache in einem Sprachkontakt besonders anfällig, er wird als Erstes abgebaut bzw. kann durch 
die Umgebungssprache leicht beeinflusst werden - Prozesse, die zum unvollständigen Spracherwerb hinzukommen und im herkunftssprachlichen Unterricht mitberücksichtigt werden müssen. Untersuchungen zu Wortfindungsaufgaben zeigen, dass bereits bei der ersten Migranten-Generation Attritionsprozesse im lexikalischen Bereich stattfinden (vgl. dazu Anstatt 2011: 5). Viel schwieriger ist dagegen, lexikalische Attritionsprozesse in der zweiten Generation nachzuweisen, denn der unvollständige Spracherwerb der Lexik ist von der lexikalischen Attrition schwer abzugrenzen. Die Untersuchungen von Anstatt zur Attrition in der zweiten Migranten-Generation legen nahe, dass die Attritionsprozesse mit der Frequenz der Lexik einhergehen: Die infrequente Lexik werde in der zweiten Generation nach mehreren Jahren Aufenthalt in Deutschland vergessen, während die frequente Lexik sich relativ stabil zeige (ebd.). Allerdings wird vermutlich in der Arbeit nicht zwischen rezeptiven und produktiven Ausprägungen der Attrition unterschieden, sodass nicht klar wird, ob die Probanden die Bedeutung der untersuchten infrequenten Lexeme z.B. verstehen, aber selbst nicht gebrauchen können.

Somit wären die systematische Arbeit an der Erweiterung des Wortschatzes aus allen funktionalen Stilen (und insbesondere aus den Stilen der konzeptionellen Schriftlichkeit: Wissenschafts-, Verwaltungsstil, publizistischer, offizieller Stil) sowie das Einbeziehen der infrequenten Lexik in den herkunftssprachlichen Russischunterricht als die wichtigsten Bedarfsbereiche auf der lexikalischen Ebene zu nennen.

Den einzelnen Bedarfsbereichen können verschiedene Ursachen zu Grunde liegen. Vom unvollständigen Spracherwerb zu trennen sind diejenigen Abweichungen, die auf die Einflüsse aus dem Deutschen und auf den deutsch-russischen Sprachkontakt zurückgeführt werden können. Ein sprachkontaktbedingter Bedarfsbereich auf der lexikalischen Ebene ist der Bereich der lexikalischen Kollokation. Laut Karl (2012) gehören zu den häufigsten Transferenzerscheinungen die Transferenz der lexikalischen Bedeutungen und der lexikalischen Kollokationen aus dem Deutschen. Ein

Anastasia Drackert und Katrin Bente Karl (Hg.), Didaktik der slawischen Sprachen

Beiträge zum 2. Arbeitskreis in Innsbruck (19.02.-20.02.2018)

(C) 2019 innsbruck university press, ISBN 978-3-903187-80-1, DOI 10.15203/3187-80-1 
paar Beispiele aus der Arbeit: *напугать яйи statt охладить '(Eier) abschrecken', *нога уснула statt затекла '(Bein) eingeschlafen', *найти термин statt договориться о встрече 'einen Termin finden', *делать практику statt проходить практику 'ein Praktikum machen', *чайный мешок statt чайный пакетик 'Teebeutel'. Daher sind Übungen zu Kollokationen, an der Universität vor allem zu typischen Kollokationen aus der Wissenschaftssprache und anderen funktionalen Stilen der konzeptionellen Schriftlichkeit, sinnvoll.

\subsection{Bedarfsbereiche auf der syntaktischen Ebene und Interpunktion}

Auf der syntaktischen Ebene ist der sprachkontaktbedingte Bedarfsbereich die syntaktische Valenz. Die Veränderung der semantischen und syntaktischen Valenz im russisch-deutschen Sprachkontakt bei der ersten und zweiten Migrantengeneration ist der Untersuchungsgegenstand der Arbeit von Wald (2009). In dieser Arbeit werden Valenzabweichungen in mündlich geführten Interviews mit ProbandInnen der ersten und zweiten Migrantengeneration untersucht. Die Autorin unterscheidet einerseits zwischen transferbedingten und nicht transferbedingten syntaktischen Abweichungen und andererseits semantischen Abweichungen. Die transferbedingten syntaktischen Abweichungen sind den Strukturen der Kontaktsprache Deutsch geschuldet, vgl. das Beispiel aus Wald (ebd.: 41) *Эmom вопрос можно ответить 'Man kann diese Frage beantworten'. Bei den nicht transferbedingten syntaktischen Abweichungen bestehen dagegen andere, teilweise nicht transparente Gründe, vgl. das Beispiel aus Wald (ebd.: 54) *[...] может из-за этого зависит 'vielleicht hängt es damit zusammen'. Auch im syntaktischen Valenzbereich - so Wald (ebd.: 31) kann man zwischen schriftlichen und mündlichen Valenzvarianten eines Verbs unterscheiden. Wenn das Wörterbuch von Apresjan/Páll (1982) beim Verb govorit' 'sprechen' nur ein Argument mit der Präposition $o$ 
'über' erfasst, besteht neben dieser standardsprachlichen Variante die im mündlichen Korpus weit verbreitete Struktur-Variante mit pro 'über'. Die letzte Struktur kommt vermehrt in der Sprache der HerkunftssprecherInnen vor (Beispielsatz aus dem Korpus von Wald): Net, ja ne znal pro če s nimi govorit' [...]. 'Nein, ich wusste nicht, worüber ich mit ihnen sprechen soll $[\ldots]^{\prime}$.

Der Valenzbereich erstreckt sich einerseits auf die lexikalische Ebene und umfasst lexikalische Kollokationen, andererseits auf die morphologische und syntaktische Ebene und berührt die Kategorie Kasus und Kasusrektion. Neben der Unsicherheit in den lexikalischen Kollokationen und neben der Instabilität des Kasussystems sind in der Herkunftssprache mündlich geprägte Valenzstrukturen dominant. Daher wird hier die syntaktische Valenz als erster Bedarfsbereich des herkunftssprachlichen Unterrichts auf der syntaktischen Ebene bestimmt.

Ein weiterer Bedarfsbereich auf der syntaktischen Ebene ist der zusammengesetzte Satz selbst. Bekanntlich kommen in der gesprochenen Sprache fast ausschließlich Elementarsätze vor und kaum zusammengesetzte Sätze. Nicht überraschend ist daher, dass die HerkunftssprecherInnen in schriftlichen Arbeiten im schreibbezogenen Unterricht bei zusammengesetzten Konstruktionen einige Probleme mit Satzverknüpfungen aufweisen. Die wichtigsten Typen des zusammengesetzten Satzes (Konditional-, Kausal-, Konzessivsatz usw.) und die entsprechenden sprachlichen Mittel (die Wahl adäquater Konjunktionen) sowie die typische Wortstellung im Haupt- und Nebensatz sollten im schriftsprachbezogenen Unterricht besonders geübt werden. Eine ausführliche Darstellung der einzelnen Bedarfsbereiche und einen sinnvollen curricularen Entwurf für den Erwerb der Kompetenzen in der schriftlichen Sprachproduktion (in der konzeptionellen Schriftlichkeit) im herkunftssprachlichen Russischunterricht macht Kukla (i.V.). 
Schließlich hängt hiermit der letzte Bedarfsbereich auf der syntaktischen Ebene zusammen: die Kenntnisse der Zeichensetzung, z.B. bei Aufzählungen, wiederholenden Konjunktionen, Komma-Regeln bei Partikeln (vvodnye slova), bei $i$ 'und' in zusammengesetzten Sätzen; Verwendung des Doppelpunktes und der Anführungszeichen.

\subsection{Bedarfsbereiche auf der Textebene}

Die Entwicklung der Textkompetenz ist gemäß Bergmann (2014: 88f) mit dem bewussten Umgang mit Sprache und der sprachlichen Analyse der die Textualität erzeugenden Mittel verbunden, z.B. der Konnektoren wie textordnende Partikel во-первых 'erstens', во-вторых 'zweitens', прежде чем 'vor allem', aber auch mit der Komposition des Textes selbst. Weiterhin bedürfen wir für das erfolgreiche Verstehen der Texte sogenanntes Musterwissen über verschiedene Textsorten und ihre stilistischen Besonderheiten: Musterwissen über ein Rezept, ein Märchen, die Nachrichten oder einen wissenschaftlichen Aufsatz usw. Hat man die Textkompetenz in der eigenen Muttersprache erworben, kann man bestimmte Wissensbereiche in die zu lernende Fremdsprache transferieren (vgl. ebd.). Transferierbar sind Bereiche z. B. der Kohärenz, Textualisierung; nicht transferierbar sind dagegen adäquate Formulierungen, stilistische Kompetenz und die einzelsprachlichen, die Textualität erzeugenden sprachlichen Mittel.

Für die HerkunftssprecherInnen, deren Herkunftssprache nur auf die mündliche Alltagssituation und daher auf mündliche Texte eingestellt ist, ist die Arbeit an der Textkompetenz von enormer Bedeutung. Daher werden als wichtigste Bedarfsbereiche auf der Textebene folgende formuliert: die Erweiterung der Textkompetenz auf verschiedene (insbesondere 
schriftsprachliche) Textsorten und das Sich-Bewusstmachen der stilistischen Differenziertheit sowie der Gebrauch der adäquaten sprachlichen Mittel. In diesem Zusammenhang können z.B. Aufgaben zu verschiedenen Textsorten wie Anzeige, Erklärung, Brief, Blog, Rezension, Reisebericht, Wissenschaftssprache gestellt werden. Weiterhin können spezielle sprachliche Mittel (Lexik und feste Wortverbindungen) eingeführt werden, die zur Argumentation, Zustimmung oder Ablehnung, Ausdruck der eigenen Meinung usw. gebraucht werden und auch eine textordnende und textkompositionale Funktion haben. Zum Bewusstmachen der stilistischen Differenziertheit können verschiedene Texte zum Vergleich angeboten werden, z.B. offizielle Texte, Wissenschaftstexte im Vergleich zu umgangssprachlichen Texten o. ä.

Abschließend werden in der folgenden Übersicht (Tabelle 1) die einzelnen Bedarfsbereiche aus systemlinguistischer Perspektive zusammengefasst.

\begin{tabular}{|l|l|}
\hline $\begin{array}{l}\text { Sprachliche } \\
\text { Ebene }\end{array}$ & $\begin{array}{l}\text { Bedarfsbereiche im herkunftssprachlichen Unterricht } \\
\text { aus systemlinguistischer Perspektive }\end{array}$ \\
\hline $\begin{array}{l}\text { phonetische } \\
\text { Ebene }\end{array}$ & $\begin{array}{l}\text { Artikulation und Rezeption einzelner Laute; } \\
\text { Laut-Schrift-Asymmetrie und der phonetischen Kombina- } \\
\text { torik (Reduktion, Assimilation, Stimmtonverlust); } \\
\text { Palatalisiertheit und Velarisiertheit der Konsonanten }\end{array}$ \\
\hline $\begin{array}{l}\text { morphematische } \\
\text { Ebene }\end{array}$ & \begin{tabular}{l} 
Sich-Bewusstmachen der Morphemik (auch der ungebun- \\
denen Morphemik); \\
Verwendung der Morphemik in Wortbildung; \\
Prinzip der Morphemkonstanz; \\
\hline $\begin{array}{l}\text { morphologische } \\
\text { Ebene }\end{array}$
\end{tabular} $\begin{array}{l}\text { Stabilisierung der unvollständig erworbenen, fehlenden } \\
\text { grammatischen Kategorien }\end{array}$ \\
\hline
\end{tabular}

Anastasia Drackert und Katrin Bente Karl (Hg.), Didaktik der slawischen Sprachen

Beiträge zum 2. Arbeitskreis in Innsbruck (19.02.-20.02.2018)

(C) 2019 innsbruck university press, ISBN 978-3-903187-80-1, DOI 10.15203/3187-80-1 


\begin{tabular}{|l|l|}
\hline $\begin{array}{l}\text { lexikalische } \\
\text { Ebene }\end{array}$ & $\begin{array}{l}\text { Erweiterung des Wortschatzes aus allen funktionalen Sti- } \\
\text { len; } \\
\text { Lexikalische Kollokationen }\end{array}$ \\
\hline $\begin{array}{l}\text { syntaktische } \\
\text { Ebene }\end{array}$ & $\begin{array}{l}\text { Syntaktische Valenzstrukturen; } \\
\text { Typen des zusammengesetzten Satzes, Konjunktionen und } \\
\text { Wortstellung; } \\
\text { Interpunktion }\end{array}$ \\
\hline Textebene & $\begin{array}{l}\text { Erweiterung der Textkompetenz auf verschiedene Textsor- } \\
\text { ten; } \\
\text { Sich-Bewusstmachen der stilistischen Differenziertheit; } \\
\text { Gebrauch der adäquaten, textkompositionalen sprachlichen } \\
\text { Mittel }\end{array}$ \\
\hline
\end{tabular}

Tab. 1 Überblick über die einzelnen Bedarfsbereiche auf verschiedenen sprachlichen Ebenen

\section{Zusammenfassung und weitergehende Anmerkungen}

Im Fokus der vorliegenden Arbeit steht die allgemeine Beschaffenheit der russischen Herkunftssprache, und es werden die Bedarfsbereiche erfasst, die aus dieser Beschaffenheit für den herkunftssprachlichen Unterricht resultieren (vgl. Tabelle 1, Abschnitt 2.6.). Dabei wird festgestellt, dass das zentrale Charakteristikum der Herkunftssprache ihr mündlicher Erwerb ist, der wiederum wie ein roter Faden durch die ganzen sprachlichen Ebenen und Phänomene verfolgt werden kann. Somit begründet der mündliche 
Modus des Spracherwerbs die Unvollständigkeit der Herkunftssprache selbst. Nebenbei sei erwähnt, dass methodische Fragen nach der Abgrenzung zwischen dem unvollständigen Erwerb einerseits und der Sprachattrition andererseits nicht im Zentrum dieser Arbeit stehen. Aus den Bereichen, die unvollständig erworben, vom Deutschen beeinflusst oder attriiert werden, geht schließlich hervor, wie die Herkunftssprache weiter erworben werden soll: vervollständigt, von Einflüssen „befreit“ und wieder erinnert.

Allgemein gesagt, können die weiteren Erwerbsziele im gesteuerten herkunftssprachlichen Unterricht auf folgende Weise zusammengefasst werden: 1. Erwerb der geschriebenen Sprache (Orthographie; in ihrer syntaktischen und stilistischen Ausdifferenzierung, Textgestaltung und Interpunktion); 2. Vervollständigung der unvollständig erworbenen morphologischen Kategorien und Stabilisierung der Kategorien; 3. Arbeit an der morphematischen Blindheit (Bewusstmachung der Morphemik) und 4. Erweiterung des lexikalischen Wortschatzes.

Schließlich soll hier noch einmal hervorgehoben werden, dass die einzelnen Bedarfsbereiche des herkunftssprachlichen Russischunterrichts zum Teil für verschiedene Lerner-Gruppen gelten können, also auch in einigen Punkten für FremdsprachenlernerInnen, und dass sie nicht allein auf die HerkunftssprecherInnen beschränkt sind. Allerdings sind sie für die HerkunftssprecherInnen in ihrer hier dargelegten Form einschlägig.

\section{Literatur}

Anstatt, T. (2011). Sprachattrition. Abbau der Erstsprache bei russischdeutschen Jugendlichen. Wiener Slawistischer Almanach 67, 7-31. Apresjan, Ju. D. \& Páll, Ė. (1982). Russkij glagol - vengerskij glagol. 
Upravlenie i sočetaemost'. Budapešt.

Bergmann, A. (Hrsg.) (2014). Fachdidaktik Russisch. Eine Einführung. Tübingen.

Bergmann, A. \& Brüggemann, N. (i. V.). Grundlagen und Prinzipien der Entwicklung eines Curriculums für herkunftssprachliche Lerner. In A. Bergmann \& N. Brüggemann (Hrsg.), Russisch als Herkunftssprache in Deutschland unterrichten. Zu linguistischen Grundlagen und didaktischen Perspektiven des Lehrens und Lernens.

Brüggemann, N. (2016). Herkunftssprache Russisch. Unvollständige Grammatik als Folge mündlichen Spracherwerbs. In A. Bazhutkina \& B. Sonnenhauser (Hrsg.), Linguistische Beiträge zur Slavistik. XXII. JungslavistInnen-Treffen in München (37-58).

Brüggemann, N. (2018). Arbeit mit SchreibanfängerInnen im herkunftssprachlichen Russischunterricht. In A. Bergmann, O. Caspers \& W. Stadler (Hrsg.), Didaktik der slawischen Sprachen-Beiträge zum 1. Arbeitskreis in Berlin (12.-14.09.2016) (177-192). Innsbruck.

Brüggemann, N. (i.V.). Zur (In-)Stabilität der grammatischen Kategorie in der Herkunftssprache Russisch (am Beispiel der Substantivkategorien Numerus, Genus und Kasus). In A. Bergmann \& N. Brüggemann (Hrsg.), Russisch als Herkunftssprache in Deutschland unterrichten. Zu linguistischen Grundlagen und didaktischen Perspektiven des Lehrens und Lernens.

Karl, K. B. (2012). Bilinguale Lexik. Nicht materieller lexikalischer Transfer als Folge der aktuellen russisch-deutschen Zweisprachigkeit. München.

Kukla, Ju. (i.V.). Syntaktische Besonderheiten in der schriftlichen Produktion von Herkunftssprechern. Analyse der Übungen in Lehrwerken und die Behandlung der syntaktischen Schwierigkeiten im Unterricht. In A. Bergmann \& N. Brüggemann (Hrsg.), Russisch als Herkunftssprache in Deutschland unterrichten. $\mathrm{Zu}$ linguistischen Grundlagen und didaktischen Perspektiven des Lehrens und Lernens.

Anastasia Drackert und Katrin Bente Karl (Hg.), Didaktik der slawischen Sprachen

Beiträge zum 2. Arbeitskreis in Innsbruck (19.02.-20.02.2018)

(C) 2019 innsbruck university press, ISBN 978-3-903187-80-1, DOI 10.15203/3187-80-1 
Polinsky, M. (1997). American Russian: Language Loss Meets Language Acquisition. In W. Browne et al. (Hrsg.), Annual Workshop on Formal Approaches to Slavic Linguistics. The Cornell Meeting 1995 (370-406). Ann Arbor.

Polinsky, M. \& Kagan, O. (2007). Heritage Languages: In the 'Wild' and in the Classroom. Language and Linguistics Compass 1/5, 368-395.

Riehl, C. M. (2014). Mehrsprachigkeit. Eine Einführung. Darmstadt.

Wald, V. (2009). Valenzstrukturen im russisch-deutschen Sprachkontakt. (Unveröffentlichte Magisterarbeit). Regensburg.

Zemskaja, E. (Hg.) (1973). Russkaja razgovornaja reč'. Moskva.

Zemskaja, E., Kitajgorodskaja, M. \& Širjaev, E. (Hrsg.) (1981). Russkaja razgovornaja reč'. Obščie voprosy. Slovoobrazovanie sintaksis. Moskva.

Zemskaja, E. (Hg.) (1983). Russkaja razgovornaja reč’. Fonetika. Morfologija. Leksika. Žest. Moskva. 
